\title{
Variación estacional de 25-hidroxi-vitamina D3, hormona paratiroidea y fosfatasa alcalina en niños escolares
}

\author{
Seasonal variations in 25-hydroxy vitamin D3, parathormone and alkaline phosphatase \\ in school-aged children
}

\author{
Gonzalo Domínguez-Menéndez ${ }^{\mathrm{a}}$, Helena Poggi ${ }^{\mathrm{a}}$, Rosario Moore ${ }^{\mathrm{a}}$, Ivonne D’Apremont ${ }^{\mathrm{a}}$, Mónica Arancibia ${ }^{\mathrm{b}}$, \\ Hernán García a, Dafne Segalla, Fidel Allendec, Sandra Solaric ${ }^{c}$, Alejandro Martínez-Aguayo ${ }^{a}$
}

\author{
aDivisión de Pediatría, Escuela de Medicina, Pontificia Universidad Católica de Chile. Santiago, Chile \\ bServicio de Pediatría, Hospital Higueras. Talcahuano, Chile \\ 'Departamento de Laboratorios Clínicos, Escuela de Medicina, Pontificia Universidad Católica de Chile. Santiago, Chile
}

Recibido: 15 de abril de 2020; Aceptado: 10 de agosto de 2020

¿Qué se sabe del tema que trata este estudio?

La deficiencia de vitamina $\mathrm{D}$ es una condición frecuente, especialmente durante los meses más oscuros en las regiones más extremas del mundo, que puede tener consecuencias graves en la salud ósea, así como manifestaciones en otros sistemas.

\section{¿Qué aporta este estudio a lo ya conocido?}

Casi un 50\% de los niños en edad escolar presentan deficiencia de vitamina D en zonas con latitud no extrema, como Santiago de Chile, con una disminución significativa de las concentraciones de 25-OH-Vitamina D en comparación al verano.

\section{Resumen}

El principal rol de la vitamina $\mathrm{D}$ es la regulación del metabolismo del calcio, cuya principal fuente es la vitamina D3 que se obtiene principalmente por la acción de la luz ultravioleta (UV) en la piel. Objetivo: Evaluar las diferencias estacionales en las concentraciones de 25-hidroxi-vitamina D3 (25OHVitD3), hormona paratiroidea (PTH), fosfatasa alcalina (FA) y calcio en niños en edad escolar. Sujetos y Método: Se midieron las concentraciones de 25OHVitD3, PTH, FA y calcio en niños de 5 a 8 años, sin suplementación de Vitamina D, reclutados en Santiago de Chile (latitud -33.4372) en distintas estaciones del año. El estatus de VitD fue definido como suficiente con concentraciones de $25 \mathrm{OHVitD} 3>20 \mathrm{ng} / \mathrm{mL}(50 \mathrm{nmol} / \mathrm{L})$, insuficiente $12-20 \mathrm{ng} / \mathrm{mL}(30-50 \mathrm{nmol} / \mathrm{L})$ y deficiente $<12 \mathrm{ng} /$ $\mathrm{mL}(30 \mathrm{nmol} / \mathrm{L})$ en base a las recomendaciones del grupo de expertos del "Consenso Global para la Prevención y Manejo del Raquitismo Nutricional”. Resultados: En total participaron 133 niños (89 prematuros menor o igual a 32 semanas), 41 durante el verano, 28 en otoño, 35 en invierno y 29 en primavera. La diferencia de las medias entre el verano y el invierno fue de $9,6 \mathrm{ng} / \mathrm{mL}$ para $25 \mathrm{OHVitD} 3$ $(\mathrm{p}<0,0001),-11,1 \mathrm{pg} / \mathrm{mL}$ para PTH $(\mathrm{p}<0,0001)$ y $-47,5 \mathrm{UI} / \mathrm{mL}$ para FA $(\mathrm{p}=0,01)$. En las concentra-
Palabras clave: Paratohormona, 25OHVitD3; Fosfatasas Alcalinas; Índice UV; Estaciones del año 
ciones de calcio no se observaron diferencias. El 97,6\% de los sujetos fueron clasificados con estatus de suficiencia ( $>20 \mathrm{ng} / \mathrm{mL}$ ) en verano, lo que disminuyó significativamente en invierno (54,3\%, $\mathrm{p}<0,0001)$. Conclusiones: Las concentraciones de 25OHVitD3 disminuyeron en aproximadamente la mitad de los niños durante el invierno, lo que se vio acompañado de un aumento de la PTH y FA, asociado a concentraciones normales de calcio. De acuerdo a nuestros resultados, la suplementación con VitD en niños podría ser necesaria durante otoño e invierno.

\section{Abstract}

The main role of Vitamin D is to regulate calcium metabolism, whose main source is vitamin D3 obtained mostly from the action of ultraviolet (UV) light on the skin. Objective: To evaluate the seasonal differences in the concentrations of 25-hydroxy-vitamin D3 (25OHVitD3), parathyroid hormone (PTH), alkaline phosphatase (ALP), and calcium in school-age children. Subjects and Method: The concentrations of 25OHVitD3, PTH, ALP, and calcium were measured in children from Santiago, Chile (latitude -33.4372), aged 5 to 8 years, without Vitamin D supplementation, in different seasons of the year. VitD status was defined as sufficient with concentrations of $25 \mathrm{OHVitD} 3>20 \mathrm{ng} / \mathrm{mL}$ ( 50 $\mathrm{nmol} / \mathrm{L})$, insufficient $12-20 \mathrm{ng} / \mathrm{mL}(30-50 \mathrm{nmol} / \mathrm{L})$ and deficient $<12 \mathrm{ng} / \mathrm{mL}(30 \mathrm{nmol} / \mathrm{L})$ based on the recommendations of the expert group of the "Global Consensus for the Prevention and Management of Nutritional Rickets". Results: 133 children participated ( 89 preterms under or equal to 32 weeks), 41 during summer, 28 in fall, 35 in winter, and 29 in spring. The difference of means between summer and winter was $9.6 \mathrm{ng} / \mathrm{mL}$ for 25OHVitD3 ( $\mathrm{p}<0.0001),-11.1 \mathrm{pg} / \mathrm{mL}$ for PTH $(\mathrm{p}<0.0001)$, and $-47.5 \mathrm{IU} / \mathrm{mL}$ for ALP $(\mathrm{p}=0.01)$. There were no differences in calcium concentrations. In summer, $97.6 \%$ of the subjects were classified with sufficiency status $(>20 \mathrm{ng} / \mathrm{mL})$, which decreased significantly in winter to $54.3 \%$ ( $p<0.0001)$. Conclusions: In winter, 25OHVitD3 concentrations decreased in approximately half of the children, which was associated with an increase in PTH and ALP, and normal calcium concentrations. According to our results, children may need VitD supplementation during fall and winter.

\section{Keywords:}

Parathyroid Hormone; 25OHVitD3;

Alkaline Phosphatase; UV Index;

Seasons

\section{Introducción}

La Vitamina D (VitD), que incluye las formas D2 y D3, es uno de los principales factores que estimula la absorción de calcio a nivel intestinal y mantiene el balance entre calcio y fosfato. Al déficit de VitD se le han adjudicado roles en enfermedades tanto agudas como crónicas, por ejemplo, pre-eclampsia, trastornos autoinmunes, enfermedades infecciosas, enfermedades cardiovasculares, cánceres y diabetes tipo 2, entre otras ${ }^{1}$.

La Vitamina D3 o colecalciferol es sintetizada en la piel en respuesta a la exposición a la luz ultravioleta (UV), y es la fuente más importante de VitD, especialmente en los meses de verano. También puede tener origen animal, principalmente en peces con alto contenido de grasas. Por otro lado, principal fuente de Vitamina D2 o ergocalciferol es vegetal ${ }^{2}$.

El metabolismo óseo está altamente regulado por varios factores, tales como VitD, hormona paratiroidea (PTH), fosfatasa alcalina (FA), calcio y fosfato. La infancia y adolescencia son períodos críticos para el establecimiento de la salud ósea para toda la vida. El déficit de VitD está asociada con una mayor secreción de PTH debido a una baja concentración de calcio sérico y 1,25- $(\mathrm{OH})_{2}$-Vitamina D3, resultando en aumento de la resorción ósea y, en consecuencia, en una disminución de la masa ósea. La deficiencia severa de VitD causa raquitismo en niños y osteomalacia en adultos. Se estima que alrededor de un billón de personas en el mundo presentan deficiencia e insuficiencia de $\mathrm{VitD}^{3}$

La síntesis de VitD3 en la piel se ve afectada por diferentes factores como la edad, la pigmentación de la piel, el uso de protector solar y la vestimenta. Como factores de riesgo de deficiencia de VitD se ha descrito obesidad, enfermedad hepática, enfermedad renal crónica o la malabsorción, insuficiencia dietética, pigmentación más oscura de la piel, nutrición parenteral a largo plazo, institucionalización, tratamiento con antiepilépticos y baja exposición solar ${ }^{2,4}$.

En los niveles de VitD también influyen variables geográficas y climáticas como latitud, altitud, estación del año y hora del día, que influyen en la síntesis de $\mathrm{VitD}^{5}$. El aumento en la prevalencia de deficiencia e insuficiencia de VitD durante el inverno se relaciona a diferentes factores, siendo la disminución de la radiación UV el más importante ${ }^{6}$. Santiago de Chile, ubicado entre los paralelos $32^{\circ} 55^{\prime}$ y $34^{\circ} 19^{\prime}$ sur, tiene cuatro estaciones marcadas durante el año, con una reducción de la radiación solar durante el otoño e invierno. Durante este período del año, el ángulo de zenit es más 
oblicuo, por lo que la absorción de radiación UV por el ozono aumenta y disminuye la que alcanza la superficie de la Tierra ${ }^{7}$. Otro factor relevante relacionado con la síntesis de la VitD es la contaminación ambiental, ya que dificulta que la radiación UV alcance la superficie de la Tierra, asociándose a una reducción de la síntesis de VitD en la piel $^{8}$. En relación a esto, existen varios reportes que muestran una mayor incidencia de deficiencia de VitD en áreas con mayor contaminación ${ }^{8,9}$.

El objetivo de este estudio es determinar si existen diferencias en la concentración de 25OHVitD3 durante las estaciones del año en escolares de Santiago de Chile, y asociarlas con concentraciones séricas de PTH, FA, y calcio, así como también con la radiación UV y contaminantes ambientales.

\section{Sujetos y Método}

\section{Diseño}

Se realizó un estudio de tipo transversal de asociación con un muestreo por conveniencia.

\section{Sujetos}

En este estudio participaron sujetos prepuberales entre 5 y 8 años en control sano en la "Red de Salud UC-Christus" y del policlínico de niños prematuros del "Complejo Asistencial Dr. Sótero del Río", muestreo que fue realizado para el proyecto FONDECYT 1160863. Se excluyeron aquellos con suplementación de VitD o con historia de patologías crónicas no resueltas.

\section{Protocolo}

Un pediatra realizó un examen físico completo a todos los sujetos en la Pontificia Universidad Católica de Chile, entre enero de 2016 y agosto de 2018. La talla fue medida utilizando un estadiómetro (Health o meter modelo $402 \mathrm{KL}$, Illinois, US) con una precisión de $0,1 \mathrm{~cm}$, y el peso fue determinado utilizando una balanza de precisión (Omron modelo HBF-510, Japón). El perímetro de cintura se midió con una cinta métrica inextensible, de acuerdo con la recomendación de la Organización Mundial de la Salud (OMS) ${ }^{10}$. La talla, índice de masa corporal (IMC) y perímetro de cintura están expresados en puntaje $Z$ de desviación estándar (puntaje-Z) de acuerdo con las referencias de la OMS.

\section{Análisis bioquímico}

En muestras de sangre tomadas después de un ayuno nocturno, se midió la concentración de 25OHVitD3 por cromatografía líquida acoplada a espectrometría de masa, así como PTH (inmunoensayo electroquimioluminiscente, Cobas, Roche), FA (Enzimático Colorimétrico, Roche, Cobas) y calcio (Colorimétrico, Roche, Cobas).
El estatus de VitD fue definido como suficiente con concentraciones de 25OHVitD3 > $20 \mathrm{ng} / \mathrm{mL}(50$ $\mathrm{nmol} / \mathrm{L})$, insuficiente $12-20 \mathrm{ng} / \mathrm{mL}$ (30-50 nmol/L) y deficiente $<12 \mathrm{ng} / \mathrm{mL}(30 \mathrm{nmol} / \mathrm{L})$ en base a las recomendaciones del grupo de expertos del "Consenso Global para la Prevención y Manejo del Raquitismo Nutricional" del año $2016^{11}$.

\section{Variables medioambientales}

El índice UV de Santiago fue obtenido de la base de datos del Servicio Meteorológico de Chile, que es responsable del registro y publicación de esta información ${ }^{12}$. En este estudio, seleccionamos los contaminantes ambientales más frecuentemente descritos en la literatura como con efecto sobre la radiación $U^{9}$ y que estuvieran disponibles del Sistema Nacional de Calidad del Aire ${ }^{13}$ : ozono $\left(\mathrm{O}_{3}\right)$, monóxido de carbono $(\mathrm{CO})$ y material particulado 10 (MP10).

\section{Análisis estadístico}

El análisis de distribución normal de las variables se realizó utilizando la prueba de Kolmogorov-Smirnov. Las variables son presentadas como medianas y rangos intercuartiles. Para las variables con distribución normal se utilizó ANOVA unidireccional y prueba de Tukey, para aquellas sin distribución normal, la prueba de Kruskal-Wallis, y posteriormente la prueba de U de Mann-Whitney (entre dos muestras independientes). Los datos fueron analizados utilizando software GraphPad ${ }^{\circledR}$ Prism version 8.0.0 para Mac OS X (GraphPad Software, San Diego, California USA).

\section{Ética}

El estudio fue aprobado por el Comité de Ética de la Facultad de Medicina de la Pontificia Universidad Católica de Chile. Uno de los padres o tutores legales firmó el consentimiento informado antes de cualquier procedimiento.

\section{Resultados}

Se reclutó un total de 147 niños, de los cuales 14 fueron excluidos. Participaron 133 niños en edad escolar (65 niñas, 48.9\%), 89 fueron muy prematuros (menor o igual a 32 semanas de edad gestacional) y 44 de término. Entre ellos no se observaron diferencias estadísticamente significativas en distribución por sexo $(\mathrm{p}=0,231)$, edad $(\mathrm{p}=0,264)$, puntaje-Z del IMC $(\mathrm{p}=0,568)$ y 25 OHVitD3 $(\mathrm{p}=0,165)$, por lo que el análisis se realizó considerando el grupo total. Las concentraciones de 25OHVitD3 medidas durante distintas épocas del año no se correlacionaron con el sexo $(\mathrm{p}=0,643)$, edad cronológica $(\mathrm{p}=0,158)$, edad gestacional $(\mathrm{p}=0,165)$, circunferencia abdominal $(\mathrm{p}=0,294)$ o puntaje-Z de IMC $(\mathrm{p}=0,545)$. 


\section{Variación estacional}

Al separar los datos de acuerdo con las estaciones del año, se observaron variaciones significativas para los promedios de concentraciones de 25OHVitD3 $(\mathrm{p}<0,0001), \mathrm{FA}(\mathrm{p}=0,001)$ y PTH $(\mathrm{p}<0,0001)$, no así en las concentraciones de calcio $(\mathrm{p}=0,356)$ (tabla 1 y figura 1). En la tabla 2 se presentas las diferencias entre el verano y las otras estaciones del año para $25 \mathrm{OH}-$ VitD3, FA y PTH.

\section{Clasificación del estatus de 25OHVitD3}

Entre los 133 sujetos, el estatus de deficiencia se observó en 2 sujetos (1,5\%), el de insuficiencia en 27 $(20,3 \%)$ y el de suficiencia en $104(78,2 \%)$. Estos tres estatus mostraron variación estacional (Chi-cuadrado de Pearson, $\mathrm{p}=0,001)$, siendo el porcentaje de suficiencia significativamente mayor en verano $(97,6 \%)$ que en otoño $(82,1 \% ; \mathrm{p}=0,0368)$, invierno $(54,3 \%$; $\mathrm{p}<0,0001)$ y primavera $(75,9 \% ; \mathrm{p}=0,0072)$.

\section{Asociación entre concentraciones de 25OHVitD3 y PTH, FA y calcio}

La concentración de 25OHVitD3 presentó una correlación inversa con la de PTH $\left(r=-0,383, \mathrm{R}^{2}=0,15\right.$, $\mathrm{p}<0,0001$, figura 2A), y ésta a su vez, se correlacionó directamente con FA $\left(r=0,240, R^{2}=0,06, p=0,0054\right.$, figura $2 \mathrm{~B})$. En este estudio no observamos una asociación estadísticamente significativa entre calcio y 25OHVitD3 ( $\mathrm{p}=0,113)$ o PTH $(\mathrm{p}=0,32)$.

\section{Asociación entre 25OHVitD3, PTH, índice UV y contaminantes ambientales}

La concentración de 25OHVitD3 fue directamente proporcional al índice UV $\left(\mathrm{r}=0,531, \mathrm{R}^{2}=0,28\right.$, $\mathrm{p}<0,0001)$ e inversamente con la concentración de CO $\left(r=-0,407, R^{2}=0,17, p<0,0001\right)$, pero no se observó asociación con MP10 ( $\mathrm{p}=0,703)$. Después de controlar por el índice UV, la asociación entre la concentración de 25OHVitD3 y CO no fue significativa $(\mathrm{p}=0,381)$. La concentración de PTH se relacionó inversamente con el índice UV $\left(r=-0,427, \mathrm{R}^{2}=0,18\right.$, $\mathrm{p}<0,0001)$ y directamente con el CO $(\mathrm{r}=0,248$, $\left.\mathrm{R}^{2}=0,06, \mathrm{p}=0,004\right)$, pero nuevamente, no se observó asociación con el MP10 ( $\mathrm{p}=0,52)$.

Las correlaciones entre las concentraciones de $25 \mathrm{OHVitD} 3$ y radiación UV de los sujetos muy prematuros y los de término fueron idénticas y no se observaron diferencias entre las pendientes $(\mathrm{F}=0,1538, \mathrm{DFn}=1$, $\mathrm{DFd}=129, \mathrm{p}=0,6956)$ (figura suplementaria 1).

Tabla 1. Características del período de recién nacidos, clínicas y bioquímicas agrupadas por estación al momento del estudio

\begin{tabular}{|c|c|c|c|c|c|}
\hline & $\begin{array}{c}\text { Primavera } \\
(n=29)\end{array}$ & $\begin{array}{l}\text { Verano } \\
(n=41)\end{array}$ & $\begin{array}{l}\text { Otoño } \\
(n=28)\end{array}$ & $\begin{array}{r}\text { Invierno } \\
(n=35)\end{array}$ & Valor $\mathbf{p}$ \\
\hline \multicolumn{6}{|c|}{ Características del período de recién nacido } \\
\hline Edad gestacional (sem) & $29[26-31]$ & $38[30-40]$ & $30[28-32]$ & $31[28-38]$ & $<0,001$ * \\
\hline Peso RN (puntaje z) & $0,06[-1,08-0,65]$ & $0,42[-0,04-1,02]$ & $0,56[-0,29-1,02]$ & $0,04[-1,09-0,87]$ & $0,02 * *$ \\
\hline Longitud de RN (puntaje z) & $-0,54[-1,60--0,03]$ & $0,34[-0,22-1,22]$ & $0,44[-0,37-0,93]$ & $-0,24[-1,58-0,62]$ & $<0,001 * *$ \\
\hline Muy prematuros (\%) & $26(89,7)$ & $16(39,0)$ & $22(78,6)$ & $25(71,4)$ & $<0,001$ \\
\hline \multicolumn{6}{|c|}{ Características clínicas al momento del análisis } \\
\hline Mujeres (\%) & $14(48,2)$ & $21(51,2)$ & $9(32,1)$ & $21(60,0)$ & 0,174 \\
\hline Edad (años) & $6,6[5,8-7,6]$ & $6,6[5,9-7,2]$ & $6,6[5,8-7,3]$ & $6,3[5,3-6,9]$ & $0,422^{*}$ \\
\hline Talla (puntaje Z) & $-0,1[-0.49-0.49]$ & $0,1[-0,59-0,66]$ & $-0,09[-0,77-0,39]$ & $-0,47[-0,86-0,47]$ & $0,466 * *$ \\
\hline IMC (puntaje Z) & $0,58[-0,6-1,53]$ & $0,58[-0,29-1,02]$ & $0,69[-0,07-1,38]$ & $0,45[-0,7-1,64]$ & $0,962 * *$ \\
\hline Perímetro abdominal $(\mathrm{cm})$ & $58,0[54,0-62,0]$ & $56,0[54,0-60,0]$ & $56,5[54,0-63,3]$ & $55,0[52,0-62,0]$ & $0,620^{*}$ \\
\hline \multicolumn{6}{|c|}{ Caracteristicas del metabolismo calcio-fósforo } \\
\hline 25OHVitD3 (ng/mL) & $26,3[21,3-30,9]$ & $30,7[26,4-34,2]$ & $24,1[21,1-26,5]$ & $21,4[17,4-25,3]$ & $<0,001 * *$ \\
\hline PTH $(p g / m L)$ & $33[29-47]$ & $24[21-30]$ & $33[29-41]$ & $35[28-45]$ & $<0,001$ * \\
\hline FA (IU/L) & $236[222-283]$ & $213[155-247]$ & $260[203-294]$ & $239[208-281]$ & $0,001 * *$ \\
\hline Calcemia (mg/dL) & $10[9,8-10,1]$ & $9,9[9,7-10,2]$ & $9,9[9,7-10,1]$ & $9,9[9,7-10,1]$ & $0,356^{*}$ \\
\hline
\end{tabular}

Los resultados están presentados como mediana y rango intercuartil. La proporción del antecedente de haber nacido muy prematuro $(<32$ semanas de gestación) y ser de sexo femenino fue comparada utilizando $\chi^{2}$ de Pearson. El valor-p fue obtenido mediante la prueba de KruskalWallis $\left(^{*}\right)$ para las variable sin distribución normal y con la prueba de Anova para las variables con distribución normal (**). 25OHVitD3: ng/ $\mathrm{mL} * 2.496=\mathrm{nmol} / \mathrm{L}$ y PTH: $\mathrm{pg} / \mathrm{mL}$ * $0.1060=\mathrm{pmol} / \mathrm{L}$. IMC, índice de masa corporal; PTH, paratohormona; FA, fofatasa alcalina. 
(A)

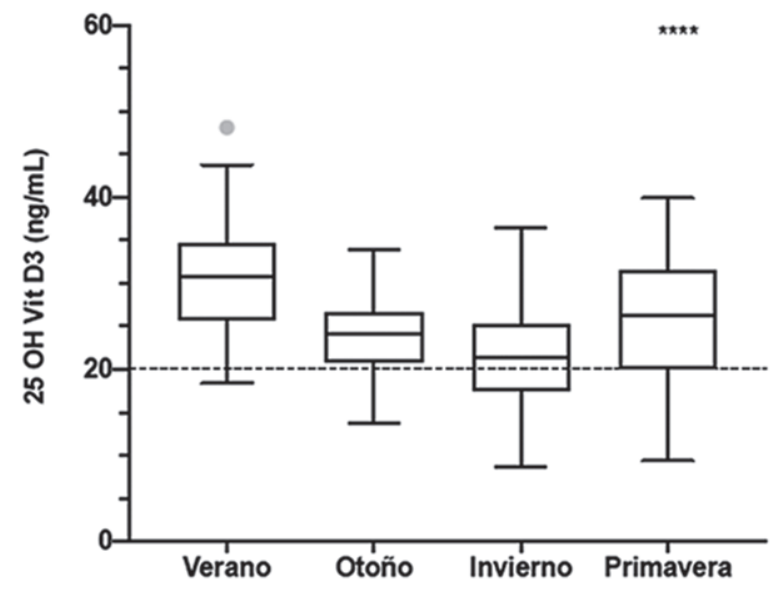

(C)

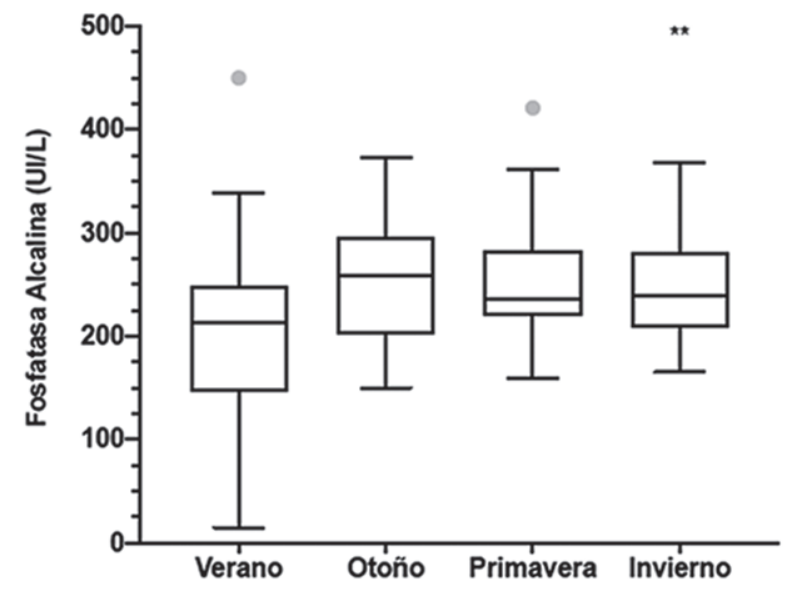

(B)

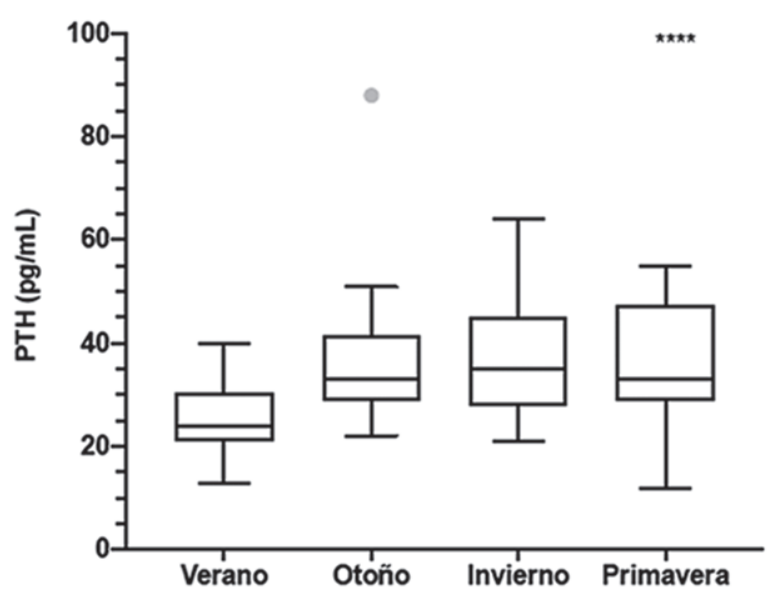

(D)

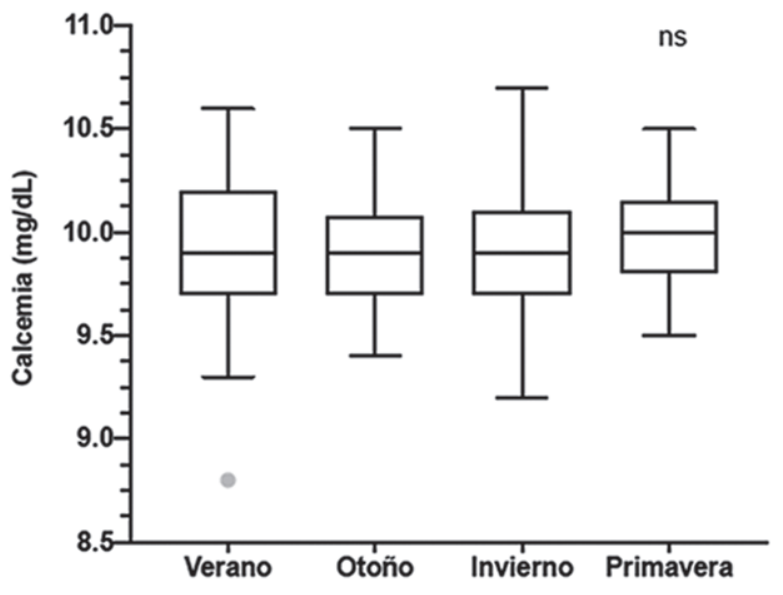

Figura 1. Concentración en las distintas estaciones y diferencias de media de concentración entre las estaciones de 25-OH-Vitamina D3 (A), PTH (B) Fosfatasa Alcalina $(\mathbf{C})$ y Calcemia (D). Los resultados están expresados como mediana y rangos intercuartiles. La línea segmentada en (A) indican el estado de Vitamina D: suficiente sobre $20 \mathrm{ng} / \mathrm{mL}$ e insuficiente/deficiente bajo $20 \mathrm{ng} / \mathrm{mL}$. FA, fosfatasa alcalina; PTH, paratohormona 25OHVitD3: $\mathrm{ng} / \mathrm{mL}$ * $2.496=\mathrm{nmol} / \mathrm{L} ; \mathrm{PTH}: \mathrm{pg} / \mathrm{mL}$ * $0.1060=\mathrm{pmol} / \mathrm{L}$.

Tabla 2. Diferencias en las concentraciones de la 250HVitD3, fosfatasas alcalinas y PTH entre el verano y las otras estaciones

\begin{tabular}{lccc}
\hline & Verano versus otoño & Verano versus invierno & Verano versus primavera \\
\hline 25OHVitD3 $(\mathrm{ng} / \mathrm{mL})$ & $6,9[2,8-11,1]^{* * *}$ & $9,6[5,7-13,5]^{* * * *}$ & $5,4[1,3-9,5]^{* *}$ \\
FA (IU/L) & $-54,7[-96,5--12,8]^{* *}$ & $-47,5[-86,8--8,2]^{* *}$ & $-49,9[-91,4--8,5]^{*}$ \\
PTH $(\mathrm{pg} / \mathrm{mL})$ & $-9,9[-12,0--5,0]^{* * *}$ & $-11,0[-14,0--7,0]^{* * * *}$ & $-10,0[-16,0--6,0] * * * *$ \\
\hline
\end{tabular}

Las diferencias en las concentraciones de la 25OHVitD3 y FA fueron analizadas con la prueba de comparación múltiple de Tukey y los resultados se muestran como diferencias de las medias, con un intervalo de confianza de $95 \%$. Las diferencias en la concentración de PTH, fueron analizadas con la prueba de Mann Whitney y los resultados se muestran como diferencias de las medianas, con un intervalo de confianza de 95\%. FA, fosfatasa alcalina; PTH, paratohormona. 
(A)

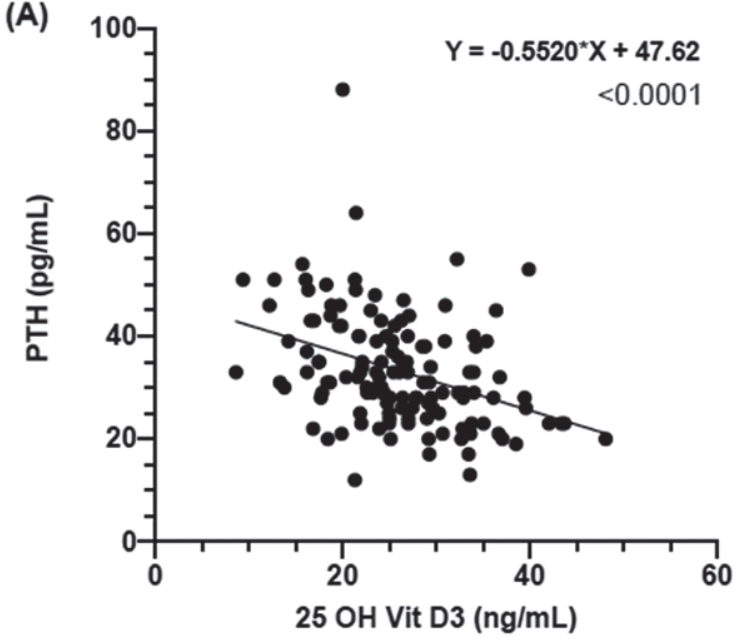

(C)

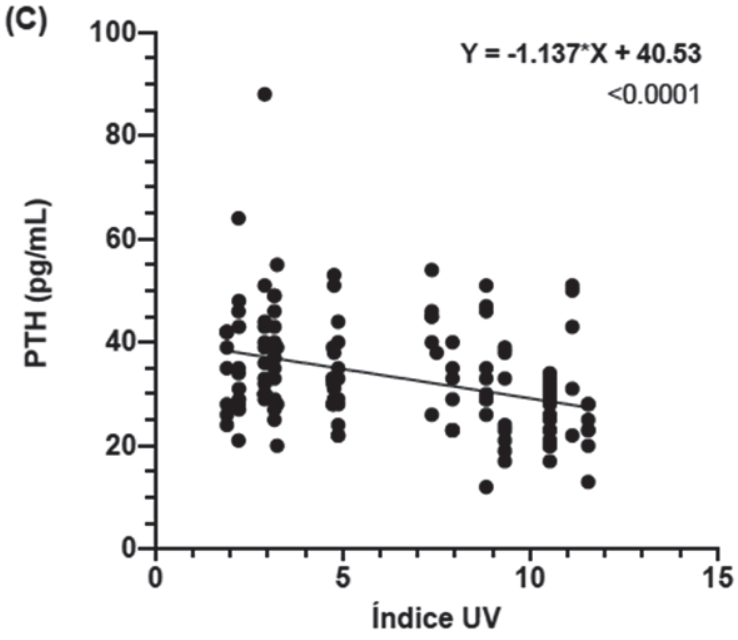

(B)
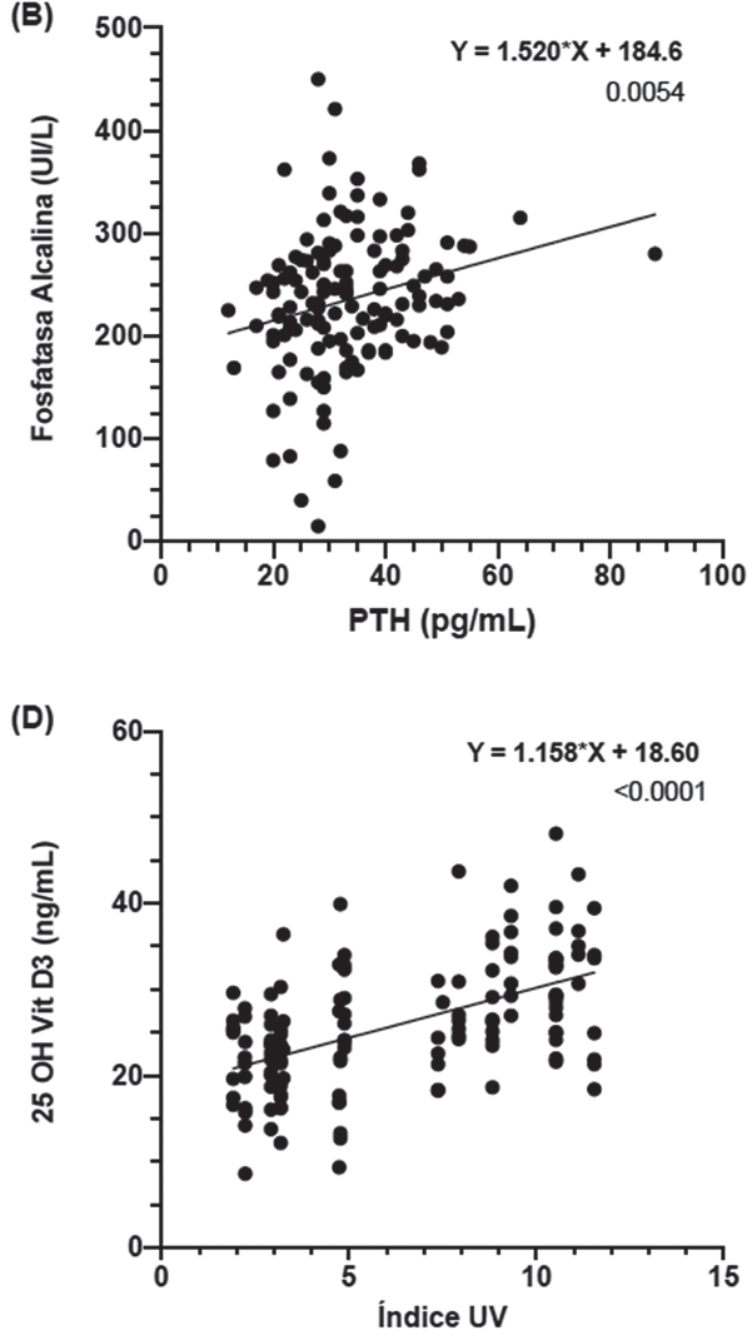

Figura 2. Asociación entre paratohormona, 25OHVitD3, fosfatasa alcalina e índice UV. FA: fosfatasa alcalina; PTH: paratohormona; índice UV: índice

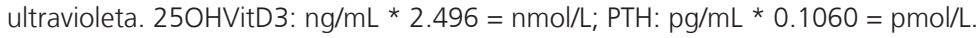

\section{Discusión}

La concentración de 25OHVitD3 mostró una variación significativa entre las distintas estaciones del año en niños prepuberales en edad escolar en Santiago de Chile y un aumento importante del porcentaje de sujetos con deficiencia en el invierno y otoño. Cabe destacar que esto se asoció a un incremento significativo en las concentraciones de PTH y FA. Estos resultados proveen evidencia para promover la discusión sobre la necesidad de suplementación de VitD durante el inverno en Chile.

En nuestro estudio no encontramos asociación entre 25OHVitD3 y edad, sexo o IMC, probablemente debido a que el grupo de estudio es muy homogéneo, ya que todos los sujetos eran prepuberales y se encon- traban dentro de un intervalo estrecho de edad, pero también podría ser explicado por el tamaño muestral. Otros estudios han mostrado diferencias en la prevalencia de deficiencia de VitD en relación a la edad, pero fueron realizados en poblaciones con un intervalo etario más amplio, incluyendo tanto niños prepuberales, adolescentes y/o adultos mayores ${ }^{14-16}$. Diferencias por sexo y estado nutricional también han sido descritas, lo que se explica por variables como la composición corporal que varía con la edad y el desarrollo puberal (en la grasa corporal se "secuestra" la VitD), características socioculturales y estilo de vida (ej. consumo de pescados, actividades al aire libre) $)^{14-25}$.

En Chile hay pocos estudios de la prevalencia de deficiencia de VitD, y sólo dos de ellos se han realizado en población pediátrica ${ }^{26,27}$. Sin embargo, es difícil 
compararlos con nuestros resultados porque se utilizaron distintas definiciones para el estatus de VitD y otros ensayos de laboratorio. En el estudio realizado en 60 niños de la ciudad de Coyhaique ( $\left.45^{\circ} 35^{\prime} \mathrm{S}\right)$, el $16,4 \%$ tenía una concentración normal de VitD (medida por radioinmunoanálisis) durante el otoño (30 a $75 \mathrm{ng} / \mathrm{mL}$ ), $20 \%$ estatus insuficiente $(20 \mathrm{a} 29 \mathrm{ng} / \mathrm{mL}$ ) y un $63,6 \%$ estatus deficiente $(<20 \mathrm{ng} / \mathrm{mL})(26) 24$ males. El otro estudio realizado en Punta Arenas (53 10 'S), en que la concentración de VitD y PTH se midió por ELISA, mostró que de los 108 niños estudiados, el $96,3 \%$ tenía deficiencia $(<20 \mathrm{ng} / \mathrm{ml})$ y $3,7 \%$ insuficiencia $(20 \text { a } 29 \mathrm{ng} / \mathrm{ml})^{27}$. Las variaciones estacionales en la concentración de VitD no sólo se observan en regiones alejadas de la línea ecuatorial, sino que también en países como Grecia, donde los niveles de VitD en niños de edad escolar fueron significativamente menores en primavera y mayores en otoño ${ }^{14}$. Resultados similares fueron observados en Dinamarca, donde también se observó mayor prevalencia de insuficiencia de VitD en primavera $^{28}$.

En base a la definición de estatus de VitD del "Consenso Global para la Prevención y Tratamiento del Raquitismo Nutricional", nuestros resultados muestran que en Santiago de Chile la mayoría de los sujetos presentan estatus de VitD suficiente $(>20 \mathrm{ng} / \mathrm{mL})$ durante el verano (97,6\%). Esto se explica probablemente por la mayor exposición solar y radiación UV que existe en este período del año en esta zona. En el invierno, sin embargo, se produce una caída importante en el porcentaje de sujetos con suficiencia $(54,3 \%)$, lo cual se debería a una disminución del índice UV y a un aumento de los contaminantes del aire en Santiago ${ }^{12,13}$. Nosotros observamos una correlación inversa entre las concentraciones de 25OHVitD3 con las de CO (no así con el MP10), pero la asociación no persiste después de controlar por la radiación UV. En consecuencia, los contaminantes del aire actuarían como un bloqueador de la radiación UV, tal como se ha descrito previamen$\mathrm{te}^{12}$.

La disminución de la concentración de $25 \mathrm{OH}-$ VitD3 que observamos durante los meses oscuros, se asoció a una respuesta fisiológica en marcadores del metabolismo óseo, tal y como se evidencia con el aumento de la PTH y consecuentemente el de las FA, con el fin de mantener una homeostasis en el calcio y fosfato. El comportamiento inversamente proporcional entre las concentraciones de $25 \mathrm{OHVitD} 3$ y PTH ha sido reportado, tanto en población adulta como pediátrica. En adultos se ha descrito que existe un plateau en la concentración de PTH con concentraciones de VitD mayores a $30 \mathrm{ng} / \mathrm{mL}(75 \mathrm{nmol} / \mathrm{L})^{22,29,30}$. Sin embargo, la concentración de VitD a partir de la cual se observa el plateau de PTH es altamente variable en población pediátrica, probablemente debido a las diferencias en sexo, color de piel y, principalmente, en la ingesta de calcio en los estudios realizados ${ }^{31,32}$. Nosotros no observamos un plateu en la asociación de 25OHVitD3 y PTH, lo que puede explicarse por la ausencia de valores extremos de VitD y por el número de sujetos de este estudio.

El costo de mantener la homeostasis del calcio por aumento de la resorción ósea podría provocar hiperparatiroidismo secundario y raquitismo hipocalcémico en niños con deficiencia importante de VitD. Si bien ningún sujeto de nuestro estudio presentó estas patologías, observamos una disminución en las concentraciones de 25OHVitD3 en invierno de casi $10 \mathrm{ng} / \mathrm{mL}$, lo que se vio acompañado de un aumento de PTH de $11 \mathrm{pg} / \mathrm{mL}$ y de 47,5 IU/L de FA, lo que sugiere un aumento de la remodelación ósea. En otras regiones del hemisferio norte se han observado incluso disminuciones aún mayores, con diferencias de media en invierno de VitD de $-12,3 \mathrm{ng} / \mathrm{mL}$ en el norte de Suecia ${ }^{33}$ y de $-15 \mathrm{ng} / \mathrm{mL}$ en Canadá ${ }^{34}$, como ejemplos. No podemos descartar que los cambios en estos marcadores del metabolismo óseo tengan un efecto negativo sobre la salud ósea, en al menos algunos niños, tal como otros autores han descrito previamente ${ }^{33,35-37}$.

Hasta la fecha no existe un consenso sobre el valor de corte para definir el estatus de VitD. Por lo tanto, la comparación entre estudios es difícil. Por ejemplo, al clasificar el estatus de deficiencia acuerdo a las definiciones del "Consenso Global para la Prevención y Tratamiento del Raquitismo Nutricional" $(<12 \mathrm{ng} / \mathrm{mL})^{11}$, de la American Academy of Pediatrics $(<15 \mathrm{ng} / \mathrm{mL})^{38}$ y de la Endocrine Society $(<20 \mathrm{ng} / \mathrm{mL})^{25}$, la frecuencia global de deficiencia de VitD en nuestra muestra sería de $1,5 \%, 5,3 \%$ y $21,8 \%$, respectivamente. Tomando en cuenta que las dos primeras recomendaciones son para población pediátrica, en nuestro estudio consideramos $<20 \mathrm{ng} / \mathrm{mL}$ como valor de corte para definir estatus de insuficiencia. Otro aspecto para la interpretación de resultados a considerar es la variedad de ensayos disponibles para medir VitD que no siempre son comparables, a pesar de los esfuerzos que se han realizado para estandarizarlos. En una comparación de distintos métodos para medir una muestra de Control de Calidad Externo con una concentración de $19 \mathrm{ng} / \mathrm{mL}$, el promedio varió entre 15 y $22 \mathrm{ng} / \mathrm{mL}$, diferencias que pueden ser aún mayores cuando se analizan muestras de pacientes ${ }^{39}$. En nuestro estudio las muestras fueron analizadas por espectrometría de masa, que es considerado el método de referencia. Sin embargo, para el uso rutinario se utilizan distintos inmunoensayos.

Dentro de las alternativas para optimizar los niveles de VitD en los meses oscuros se ha descrito la utilidad de la exposición solar. En un estudio en el Reino Unido se demostró que la exposición de cara, cuello y manos al medio día por una hora, antes de la aparición 
de eritemas en la piel, sería suficiente para mantener niveles normales de VitD, y sería equivalente a la suplementación con 400 UI de vitamina D $3^{40}$. Sin embargo, la exposición solar sin una adecuada protección de la piel tiene riesgos inmediatos y a largo plazo, tales como quemaduras y cáncer de piel.

La falta de exposición a luz UV también puede ser compensada con la suplementación de VitD, tanto de alimentos como a través de fármacos. Su suplementación universal es una práctica común en niños menores de un año de edad, pero por sobre esta edad no existe consenso. En Estados Unidos, la American Academy of Pediatrics ha recomendado desde el año 2003 la suplementación en niños y adolescentes que no consumen productos lácteos fortificados o multivitamínicos, así como en otros grupos de riesgos ${ }^{41}$ similar a lo recomendado por la European Academy of Paediatrics ${ }^{4}$ y la Endocrine Society en Estados Unidos ${ }^{25}$. Otras sociedades científicas, como el Comité de Nutrición de la European Society for Paediatric Gastroenterology, Hepatology and Nutrition (ESPGHAN) y la North American Society for Pediatric Gastroenterology, Hepatology and Nutrition (NASPGHAN), no recomiendan esta suplementación como protocolo por la falta de evidencia de su beneficio ${ }^{42}$.

En algunas regiones, como por ejemplo en Ushuaia, Argentina $\left(55^{\circ} \mathrm{S}\right)$ y en Paris, Francia $\left(48.8^{\circ} \mathrm{N}\right)$, que se encuentran en latitudes más extremas que Santiago de Chile, los niños son suplementados con 100.000 UI de Vitamina D al comienzo del inverno y 3 meses después $^{43,44}$. En el caso argentino, los 18 sujetos evaluados presentaron un nivel suficiente de VitD antes y después de la suplementación, sin presentar niveles de intoxicación. La Endocrine Society en Estados Unidos recomienda una ingesta diaria de VitD de 400 UI, 600 UI y 600 a 800 UI diarias para niños menores de un año, niños de 1 a 18 años y todos los adultos con factores de riesgo, respectivamente, de manera de prevenir la deficiencia de $\mathrm{VitD}^{25}$. En Chile no hay una normativa que rija la suplementación con VitD en niños mayores de un año. Sin embargo, de acuerdo a nuestros resultados en niños de 5 a 8 años, la suplementación podría ser necesaria durante los meses de otoño e invierno, al menos en Santiago de Chile, así como en regiones de latitudes similares o más australes, sobre todo en niños con factores de riesgo de déficit de esta vitamina.

Nuestro trabajo representa evidencia inicial a nivel local sobre la disminución de 25OHVitD3 (medida por espectrometría de masa) y su efecto sobre los marcadores de remodelación ósea en los meses de invierno. Para poder realizar recomendaciones locales de suplementación de VitD consideramos necesario realizar estudios longitudinales con un mayor número de sujetos de distintos grupos etarios.

\section{Conclusión}

En los niños prepuberales de este estudio no encontramos diferencias en las concentraciones de $25 \mathrm{OH}-$ VitD3 según sexo, edad y estado nutricional. Durante los meses con menor índice de radiación UV hay menor concentración de 25OHVitD3 y una respuesta fisiológica con aumento de PTH y FA sugerente de una mayor resorción ósea para mantener la homeostasis del calcio. En invierno la proporción de sujetos con niveles suficientes de VitD disminuyen aproximadamente a la mitad. La disminución de VitD se asoció a un menor índice de radiación UV, y los contaminantes ambientales solo se relacionaron de forma indirecta con los niveles de VitD a través de la radiación UV.

\section{Responsabilidades Éticas}

Protección de personas y animales: Los autores declaran que los procedimientos seguidos se conformaron a las normas éticas del comité de experimentación humana responsable y de acuerdo con la Asociación Médica Mundial y la Declaración de Helsinki.

Confidencialidad de los datos: Los autores declaran que han seguido los protocolos de su centro de trabajo sobre la publicación de datos de pacientes.

Derecho a la privacidad y consentimiento informado: Los autores han obtenido el consentimiento informado de los pacientes y/o sujetos referidos en el artículo. Este documento obra en poder del autor de correspondencia.

\section{Conflicto de intereses}

Los autores declaran no tener conflicto de intereses.

\section{Agradecimientos}

Estamos en deuda con los niños que han participado en nuestro estudio y sus familias. 


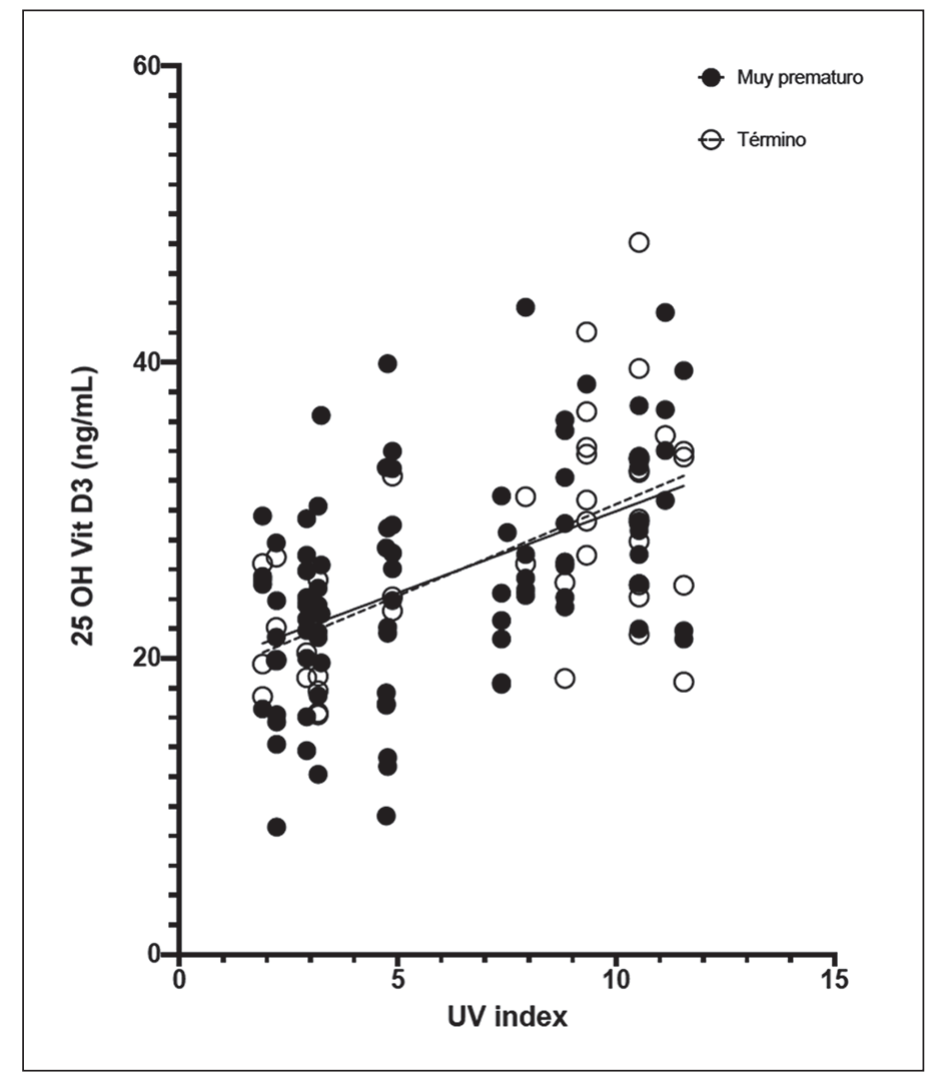

Figura Suplementaria 1. Asociación entre el índice UV y 25OHVitD3 distribuidos por el antecedente de haber nacido muy prematuro o de término. Los niños nacidos muy prematuros son representados por círculos cerrados y su relación lineal por una línea continua. Los niños nacidos de témino están representados por círculos abiertos y su regresión lineal por una línea segmentada.

\section{Referencias}

1. Holick MF. The vitamin D deficiency pandemic: Approaches for diagnosis, treatment and prevention. Rev Endocr Metab Disord. 2017;18(2):153-65.

2. Lips P. Vitamin D physiology. Prog Biophys Mol Biol. 2006;92(1):4-8.

3. Holick MF. Vitamin D: a D-Lightful health perspective. Nutr Rev. 2008;66(SUPPL.2):S182-94.

4. Grossman Z, Hadjipanayis A, Stiris T, et al. Vitamin D in European childrenstatement from the European Academy of Paediatrics (EAP). Eur J Pediatr. 2017;176(6):829-31.

5. Root AW. Disorders of Bone Mineral Metabolism: Normal Homeostasis. En: Sperling MA, editor. Pediatric Endocrinology [Internet]. 3rd Ed. W.B. Saunders; 2008 [citado 4 de septiembre de 2019]. p. 74-126. Disponible en: https:// www.sciencedirect.com/science/article/ pii/B9781416040903500089

6. Webb AR, Kline L, Holick MF. Influence of Season and Latitude on the Cutaneous Synthesis of Vitamin D3: Exposure to Winter Sunlight in Boston and Edmonton Will Not Promote Vitamin D3 Synthesis in Human Skin. J Clin Endocrinol Metab. 1988;67(2):373-8.

7. Mousavi SE, Amini H, Heydarpour P, Amini Chermahini F, Godderis L. Air pollution, environmental chemicals, and smoking may trigger vitamin D deficiency: Evidence and potential mechanisms. Environ Int. 2019;122:67-90.

8. Feizabad E, Hossein-nezhad A, Maghbooli Z, Ramezani M, Hashemian R, Moattari S. Impact of air pollution on vitamin D deficiency and bone health in adolescents. Arch Osteoporos. 2017;12(1):1-7.

9. Hoseinzadeh E, Taha P, Wei C, et al. The impact of air pollutants, UV exposure and geographic location on vitamin D deficiency. Food Chem Toxicol. 2018;113:241-54.

10. World Health Organisation (WHO). Waist Circumference and WaistHip Ratio. Report of a WHO Expert
Consultation. 2008;8-11. Disponible en: https://apps.who.int/iris/bitstream/ handle/10665/44583/9789241501491_eng. pdf?ua $=1$

11. Munns CF, Shaw N, Kiely M, et al. Global Consensus Recommendations on Prevention. J Clin Endocrinol Metab. 2016;101(2):394-415.

12. Dirección Meteorológica de Chile - Servicios Climáticos. Dirección Meteorológica de Chile [Internet]. 2019 [citado 1 de julio de 2019]. Disponible en: https://climatologia.meteochile.gob.cl/ application.

13. Ministerio del Medio Ambiente. Sistema de información nacional de calidad del aire [Internet]. 2019 [citado 1 de julio de 2019]. Disponible en: https://sinca.mma. gob.cl/index.php/estacion/index/key/D14.

14. Manios Y, Moschonis G, Hulshof T, et al. Prevalence of Vitamin D deficiency and insufficiency among schoolchildren in Greece: The role of sex, degree of urbanisation and seasonality. Br J Nutr. 2017;118(7):550-8. 
15. Basatemur E, Horsfall L, Marston L, Rait G, Sutcliffe A. Trends in the Diagnosis of Vitamin D Deficiency. Pediatrics. 2017;139(3):e20162748.

16. Ginty F, Cavadini C, Michaud P-A, et al. Effects of usual nutrient intake and vitamin D status on markers of bone turnover in Swiss adolescents. Eur J Clin Nutr. 2004;58:1257-65.

17. Okabe H, Shimizu C, Yamamoto $\mathrm{M}$, et al. Determination of serum 25-hydroxyvitamin D 3 by LC/MS/ MS and its monthly variation in Sapporo indoor workers. Anal Sci. 2018;34(9):1043-7.

18. Karagüzel G, Dilber B, Çan G, Ökten A, Değer O, Holick MF. Seasonal Vitamin D Status of Healthy Schoolchildren and Predictors of Low Vitamin D Status. J Pediatr Gastroenterol Nutr. 2014;58(5):654-60.

19. Mithal A, Wahl DA, Bonjour J-P, et al. Global vitamin D status and determinants of hypovitaminosis D. Osteoporos Int. 2009;20(11):1807-20.

20. Saqib MAN, Rafique I, Hayder I, et al. Comparison of vitamin D levels with bone density, calcium, phosphate and alkaline phosphatase - An insight from major cities of Pakistan. J Pak Med Assoc. 2018;68(4):543-7.

21. Niculescu DA, Capatina CAM, Dusceac R, Caragheorgheopol A, Ghemigian A, Poiana C. Seasonal variation of serum vitamin D levels in Romania. Arch Osteoporos. 2017;12(1).

22. Sahin ON, Serdar M, Serteser M, Unsal I, Ozpinar A. Vitamin D levels and parathyroid hormone variations of children living in a subtropical climate: A data mining study. Ital J Pediatr. 2018;44(1):1-7.

23. Tolppanen A-M, Fraser A, Fraser WD, Lawlor DA. Risk Factors for Variation in 25-Hydroxyvitamin D3 and D2 Concentrations and Vitamin D Deficiency in Children. J Clin Endocrinol Metab. 2012;97(4):1202-10.

24. Gutiérrez Medina S, Gavela-Pérez T, Domínguez-Garrido MN, et al. The influence of puberty on vitamin D status in obese children and the possible relation between vitamin D deficiency and insulin resistance. J Pediatr Endocrinol Metab. 2015;28(1-2):105-10.

25. Holick MF, Binkley NC, Bischoff-Ferrari
HA, et al. Evaluation, treatment, and prevention of vitamin D deficiency: An endocrine society clinical practice guideline. J Clin Endocrinol Metab. 2011;96(7):1911-30.

26. Le Roy C, Reyes M, González JM, Pérez-Bravo F, Castillo-Durán C. Estado nutricional de vitamina $\mathrm{D}$ en pre escolares chilenos de zonas australes. Rev Med Chil. 2013;141(4):435-41.

27. Brinkmann K, Le Roy C, Iñiguez G, Borzutzky A. Deficiencia severa de vitamina D en niños de Punta Arenas, Chile: influencia de estado nutricional en la respuesta a suplementación. Rev Chil Pediatría. 2015;86(3):182-8.

28. Hansen L, Tjønneland A, Køster B, et al. Vitamin D status and seasonal variation among danish children and adults: A descriptive study. Nutrients. 2018;10(11).

29. Touvier M, Deschasaux M, Montourcy $\mathrm{M}$, et al. Interpretation of plasma PTH concentrations according to $25 \mathrm{OHD}$ status, gender, age, weight status, and calcium intake: Importance of the reference values. J Clin Endocrinol Metab. 2014;99(4):1196-203.

30. Serdar MA, Can BB, Kilercik M, et al. Analysis of Changes in Parathyroid Hormone and $25(\mathrm{OH})$ Vitamin D Levels with Respect to Age, Gender and Season: A Data Mining Study. J Med Biochem. 2017;36(1):73-83.

31. Kang JI, Lee YS, Han YJ, Kong KA, Kim HS. The serum level of 25-hydroxyvitamin D for maximal suppression of parathyroid hormone in children: The relationship between 25- hydroxyvitamin D and parathyroid hormone. Korean J Pediatr. 2017;60(2):45-9.

32. Crews BO, Moore J, Dietzen DJ. Circulating intact parathyroid hormone is suppressed at 25-hydroxyvitamin D concentrations $>25 \mathrm{nmol} / \mathrm{L}$ in children. J Pediatr Endocrinol Metab. 2014;27(78):657-60.

33. Öhlund I, Silfverdal SA, Hernell O, Lind T. Serum 25-hydroxyvitamin D levels in preschool-age children in northern sweden are inadequate after summer and diminish further during winter. J Pediatr Gastroenterol Nutr. 2013;56(5):551-5.

34. Whiting SJ, Langlois KA, Vatanparast $\mathrm{H}$, Greene-Finestone LS. The vitamin D status of Canadians relative to the 2011 Dietary Reference Intakes: An examination in children and adults with and without supplement use. Am J Clin Nutr. 2011;94(1):128-35.

35. Greer FR. Defining vitamin D deficiency in children: Beyond 25-OH vitamin D serum concentrations. Pediatrics. 2009;124(5):1471-3.

36. Hill TR, Cotter AA, Mitchell S, et al. Vitamin D status and parathyroid hormone relationship in adolescents and its association with bone health parameters: Analysis of the Northern Ireland Young Heart's Project. Osteoporos Int. 2010;21(4):695-700.

37. Houghton LA, Szymlek-Gay EA, Gray AR, Ferguson EL, Deng X, Heath ALM. Predictors of vitamin D status and its association with parathyroid hormone in young New Zealand children. 2010 p. 69-76.

38. Misra M, Pacaud D, Petryk A, CollettSolberg PF, Kappy M. Vitamin $\mathrm{D}$ deficiency in children and its management: Review of current knowledge and recommendations. Pediatrics. 2008;122(2):398-417.

39. French D. The (Sun)Light and Dark of 25-Hydroxyvitamin D Testing. J Appl Lab Med. 2018;3(3):460-73.

40. Webb AR, Engelsen O. Ultraviolet Exposure Scenarios: Risks of Erythema from Recommendations on Cutaneous Vitamin D Synthesis. Adv Exp Med Biol. 2008;624:72-85.

41. Gartner LM. Prevention of Rickets and Vitamin D Deficiency: New Guidelines for Vitamin D Intake. Pediatrics. 2003;111(4):908-10

42. Braegger C, Campoy C, Colomb V, et al. Vitamin D in the Healthy European Paediatric Population. J Pediatr Gastroenterol Nutr. 2013;56(6):692-701.

43. Tau C, Ciriani V, Scaiola E, Acuña M. Twice single doses of 100,000 IU of vitamin $\mathrm{D}$ in winter is adequate and safe for prevention of vitamin D deficiency in healthy children from Ushuaia, Tierra Del Fuego, Argentina. J Steroid Biochem Mol Biol. 2007;103(3-5):651-4.

44. Vidailhet M, Mallet E, Bocquet A, et al. Vitamin D: still a topical matter in children and adolescents. A position paper by the Committee on Nutrition of the French Society of Paediatrics. Arch Pediatr. 2012;19(3):316-28. 